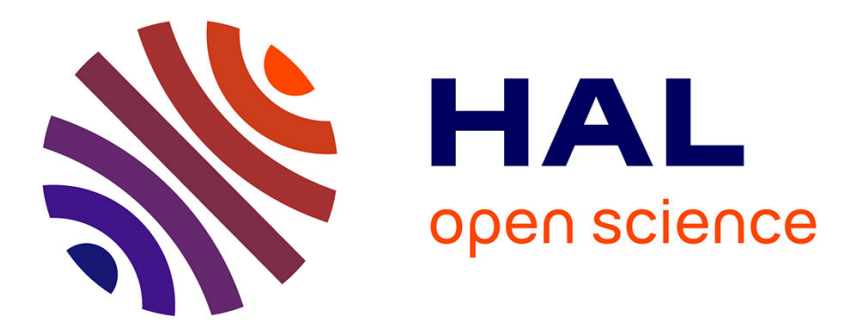

\title{
Outdoor 3D Acquisition System for Small and Fast Targets. Application to honeybee monitoring at the beehive entrance
}

\author{
Guillaume Chiron, Petra Gomez-Krämer, Michel Ménard
}

\section{To cite this version:}

Guillaume Chiron, Petra Gomez-Krämer, Michel Ménard. Outdoor 3D Acquisition System for Small and Fast Targets. Application to honeybee monitoring at the beehive entrance. GEODIFF 2013, Feb 2013, Barcelona, France. pp.10-19, 10.5220/0004346300100019 . hal-00826421

\section{HAL Id: hal-00826421 \\ https://hal.science/hal-00826421}

Submitted on 27 May 2013

HAL is a multi-disciplinary open access archive for the deposit and dissemination of scientific research documents, whether they are published or not. The documents may come from teaching and research institutions in France or abroad, or from public or private research centers.
L'archive ouverte pluridisciplinaire HAL, est destinée au dépôt et à la diffusion de documents scientifiques de niveau recherche, publiés ou non, émanant des établissements d'enseignement et de recherche français ou étrangers, des laboratoires publics ou privés. 


\title{
Outdoor 3D Acquisition System for Small and Fast Targets. Application to honeybee monitoring at the beehive entrance
}

\author{
Guillaume Chiron $^{1}$, Petra Gomez-Krämer ${ }^{1}$, and Michel Ménard ${ }^{1}$ \\ L3I, Université de la Rochelle, Avenue M. Crépeau, La Rochelle, France \\ \{guillaume.chiron, petra.gomez, michel.menard\}@univ-lr.fr
}

\begin{abstract}
In response to recent biologists needs, we propose to lay the foundations of a stereo vision-based system for monitoring honeybees in $3 \mathrm{D}$ at the beehive entrance. In this context, this article highlights difficulties and introduces some solutions for problems related to the detection of the bees and tracking them in 3D under uncontrolled conditions. This study is a prerequisite for our next step that aims to detect and analyze behaviors of the bees based on their trajectories and additional external data such as the weather or health conditions.
\end{abstract}

\section{Introduction}

The honeybee is a bio-indicator species, and nowadays researchers use beehives for environment monitoring purpose. An overdose of contaminants in the environment could result in bees excessive death-rate or behavior alterations. Behavior alterations are more and more spotted despite the difficulties of observing them at a big scale. Thus, in response to biologists' needs, it becomes essential to collect data on bees at different levels: numbers of bees, trajectories and behaviors. When done manually on videos, this process is time consuming and suffers from a lack of precision due to human errors. We believe that computer vision can effectively achieve those tasks.

Monitoring bees automatically in a outdoor uncontrolled environment arises a lot of constraints. Bees are small are fast targets and their motions could be chaotic. The high activity especially on front of the beehive results in a high occlusion rate. Working in natural conditions also raises problems such as background soiling.

Counting bees has been the first automatic application technically feasible. [1] introduced the first functionally mechanical counter. Nowadays, the less intrusive infrared sensors counters presented in [2] are widely used. A new category of counters are also able to identify bees individually by radio frequency identification (RFID) [3].

Vision based bee trajectory analysis is recently having a growing interest. The literature covers important methods for 2D tracking and behavior analysis of bees inside the beehive. In [4], bees are tracked using a hybrid particle filter. [5] proposes a method based on hidden markov models (HMM) to detect bees' behavior from their trajectories. [6] goes further by introducing a joint tracking and behavior analysis. In addition to that work, [7] adds geometric constraints and an weighted adaptive pattern to enhance the detection. 
Recently, [8] introduces a non-invasive video monitoring system that tracks bees on front of their beehive for a counting purpose. This application is based on a classic 2D vision acquisition system and uses global nearest neighbor (GNN) and interactive multiple model (IMM) comparable methods to track the multiple targets. The preliminary counting error rate results presented are still below the other counters. In [9], the authors attempted to track bees visiting flowers. This application is mono target and provides short and noisy trajectories which are not suitable for behavior analysis.

Our work takes place in the context of an environment monitoring platform illustrated by Figure 1. The main purpose of the platform is to cross data and to make it available to biologists. [10] introduced a first block: a robust vision based bee counter. A RFID version of the counter will be added in a near future thanks to a partnership (INRA institute). Blocks such as an air quality monitor are also planned to be linked to the platform. Our beehive monitoring system is one of the blocks under construction. This block could also use data from other blocks to improve their process. For example, the temperature, the time of the day and the historical data could be used to choose a adapted bee's motion model to improve the tracking.

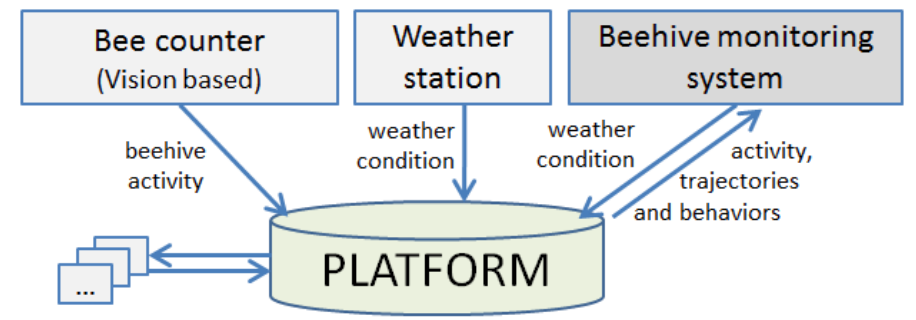

Fig. 1. Environmental monitoring platform sharing information with different blocks. This article introduces the base for the beehive monitoring system block

The purpose of our project is to analyse behaviors of honeybees on front of the beehive. We introduce in this article the base for a beehive activity monitoring system and highlight the advantages and drawbacks of using a 3D vision for that application. This article is organized as follows. We present our 3D acquisition system in Section 2. Sections 3 and 4 introduce the segmentation and the tracking methods used. Section 5 shows some preliminary results. Finally, Section 6 concludes by opening promising perspectives about tracking and behavioral analysis.

\section{Acquisition System}

We present in this section the constraints linked to the application. Then we summarize suitable 3D sensors available on the market in 2012. Finally we focus on a stereo vision system and its configuration. 


\subsection{Application Constraints}

Several constraints have to be taken into account for the choice of the 3D camera like the number, the size, the dynamic of the targets, the lighting conditions and the scene background. Each constraint is outlined in the following.

Number: Figure 2 shows an high activity on front of the beehive. The bee counter [10] reveled that bees' arrivals and departures could occur by important waves. This big amount of target evolving in 3D results in many occlusions.

Size: To assure counting purpose, the camera needs to capture the entire $50 \mathrm{~cm}$ wide board from where bees get in and out. Adult bees measure in average $12 * 6 \mathrm{~mm}$ and to be detected on the flight board, we set a limit of 6 pixels per bee on the images. Thus, XcamResolution $=(6 \mathrm{px} / 0.6 \mathrm{~cm}) * 50 \mathrm{~cm}=500 \mathrm{px}$ is the minimum resolution satisfying that small sized target constraint.

Dynamic: Bee motion is barely predictable. They can fly at speed of $8 \mathrm{~m} / \mathrm{s}$, so they could cross the whole flight board and being only captured on 1 or 2 images with a classic 24 frame/sec sensor. Even if we observed mostly slower bees around the beehive, a high frequency capturing system is recommended. An average exposure time results in blurring for wings flapping but that not important for our application.

Light: Captures are performed outdoor, so lighting conditions are not controllable. Images could contain more bees' shadows than bees themselves. The sunlight interferes with 3D sensors technologies such as infrared grid projection/sensors (e.g. Microsoft Kinect).

Background: [8] segment the bees from a white flight board which is an optimal condition. Our application aims to work on a textured flight board (e.g. due to dirt) which could become similar to bees' color.

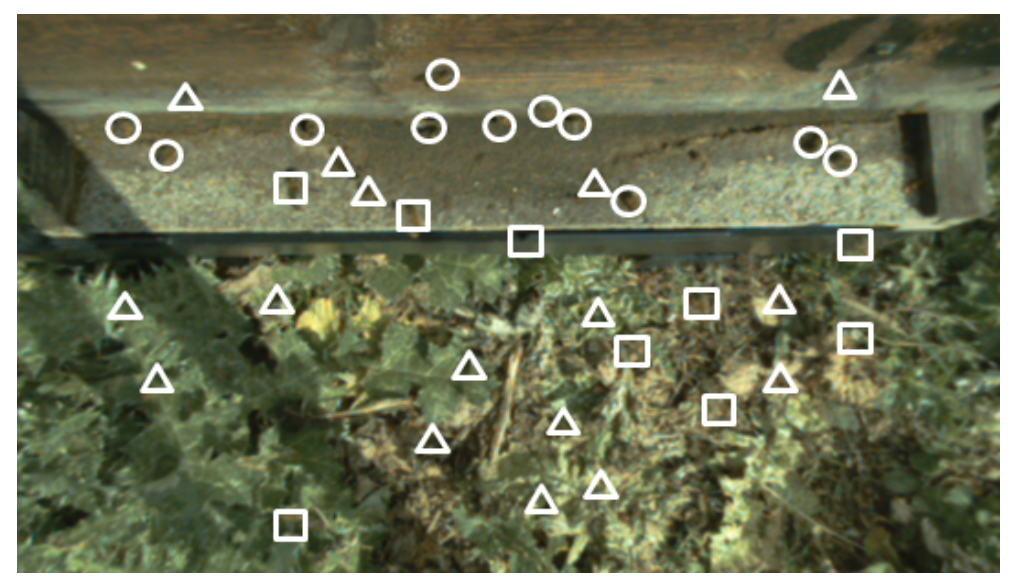

Fig. 2. Beehive entrance captured by a $2 \mathrm{D}$ camera. Circles mark 11 walking bees; squares mark 8 flying bees; triangles mark 13 bee shadows 
According to the constraints mentioned above, we believe that especially the high occlusion rate and the chaotic dynamic of the targets require another additional data (3rd dimension) to assure a robust detection and tracking of the targets.

\subsection{Candidate 3D Sensors}

We focus our attention on two kinds of 3D sensors (also called 2.5D sensors): time of flight (TOF) and stereo vision cameras. Contrary to a homemade multiple camera systems [11], those built-in 3D cameras don't require any calibration and directly provide a depth map and gray (or RGB) images corresponding. As we later focus on stereo vision systems, additional information on TOF cameras are given in [12].

Table 1. Comparison of 6 camera configurations (resolution and frame rate) proposed by leaders of TOF and stereo vision systems in 2012

\begin{tabular}{|c|c|}
\hline Time of flight & Stereo vision \\
\hline Cb. Nano (Pmdtec), 160x120px,90fps & Bum.Bee 2 (P.Grey), 640x480px,48fps \\
\hline D70 (Fotonic), 160px*120px, 50fps & G3 EV (TYZX), 752x480px, 50fps \\
\hline SR4000 (Mesa), 176px *144px, 50fps & SVC (F.Robotics), 752x480px, 30fps \\
\hline
\end{tabular}

The resolution of TOF cameras presented in Table 1 are too low for our application because the requirement of the representation of a bee by 6 pixels is not satisfied. Nevertheless, the high frame rate of the $\mathrm{Cb}$. Nano kept our attention. Capturing a fast moving object at a speed of $90 \mathrm{fps}$ would reduce tracking failures. In that case we would focus on a smaller part of the flight board to get enough pixels to detect a bee. Then, we also retain the stereo vision G3 EV that seems to satisfy the small and fast target constraints.

a) StereoCamera

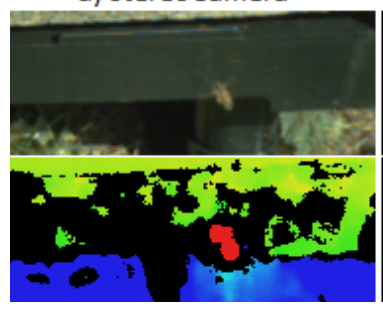

b) TOF Camera

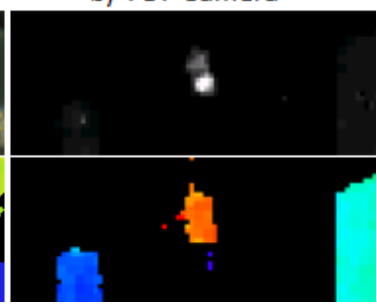

Fig. 3. Images (top row) and depth maps (bottom row) captured by Cb.Nano (TOF) and G3 EV (stereo vision) for a sample bee

Figure 3 shows some images and depth maps respectively acquired by the G3 EV and CamBoard Nano cameras. The image a) provides a clear RGB image while the image b) provides an intensity map limited by the amount of light received by the sensor. The depth map a) is well computed on highly textured areas. In reasonable 
conditions (targets closer than $50 \mathrm{~cm}$ from the camera and moving at an average speed), bees' textures are well captured and thus left and right images are correctly matched. The close detection range of $2 \mathrm{~m}$ of $\mathrm{b}$ ) filters the main part of the background. Then, depth for white or reflecting objects is not well captured. Also, targets getting far from the center tend to be not clearly captured by the sensor. Finally, stereo cameras have a lower frame rate than TOF cameras due to the depth map computation complexity. The G3 EV reaches 50 fps thanks to an embedded processors unit. Table 2 summarizes the strengths and weaknesses of both cameras tested and evaluated according to our application constraints.

Table 2. Compared capture with TOF (CamBoard Nano) and stereo vision camera (G3 EV) according to our application constraints

\begin{tabular}{|l|c|c|}
\hline & Cb. Nano & G3 EV \\
\hline Frame rate & +++ & ++ \\
\hline Resolution & + & +++ \\
\hline Small targets detection & +++ & +++ \\
\hline Fast targets detection & +++ & ++ \\
\hline Depth map accuracy & ++ & +++ \\
\hline Depth map consistency & +++ & ++ \\
\hline
\end{tabular}

So far, the G3 EV stereo camera is the best compromise to capture high resolution images and depth maps at a correct frequency.

\subsection{Stereo Camera Configuration}

Figure 4 shows the configuration of the stereo camera targeting the flight board. We choose a small baseline which is more accurate for close rang applications. Then, according to the lenses available from the constructor, we find the best solution to maximize the tracking area $\mathrm{TA}=(\mathrm{f} / 2)^{2} / \tan (\alpha / 2)$ keeping at least 6 pixels per centimeters on the flight board using (1) to be able to detect the bee correctly. Finally the chosen configuration for this application is a $3 \mathrm{~cm}$ baseline camera equipped with 62 degree HFOV lenses placed at a height of $50 \mathrm{~cm}$ from the flight board.

$$
\text { PixelPerCm }=\frac{X \text { camResolution }}{2 h \tan \left(\frac{\propto}{2}\right)}
$$

The stereo camera computes the depth image using patch matching algorithms between the right and left images. As shown in Figure 5, different effects are observable on the depth map according to the situation. The background part hidden by a target is different according to the point of view the target is observed from. For every flying target, the depth map shows the target distance as well as a depth shadow region corresponding to the unmatched background. Thus, as well as normal distance information, depth shadow areas on the depth map can witness the presence of targets. Sometimes the matching step fails and wrong patches are associated together, it results in inconsistent depth regions. 


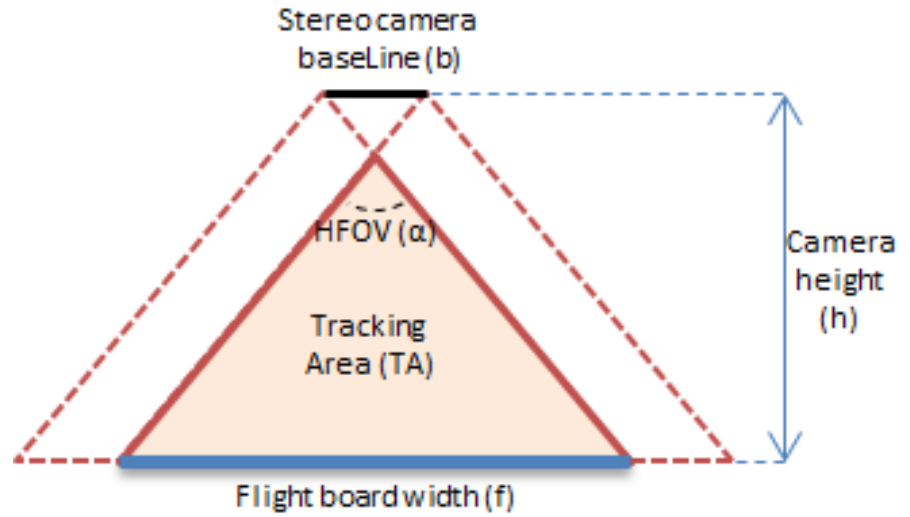

Fig. 4. Stereo camera configuration

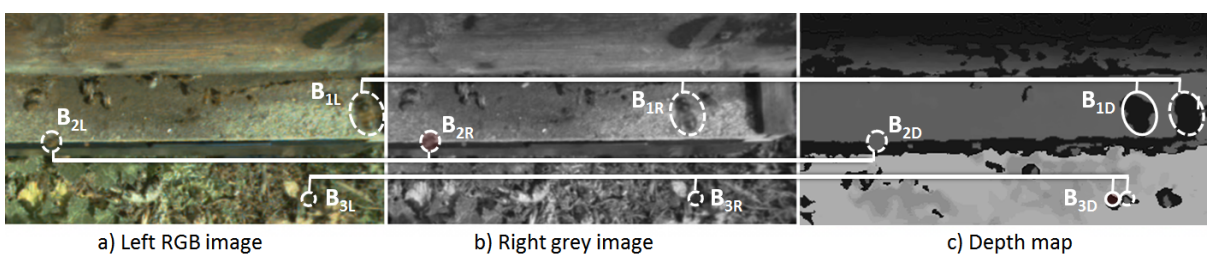

Fig. 5. Different depth map effects observed with the G3 EV camera. B (high speed target) isn't matched between a) and c), and it results in the right shadow effect on c), plus its high height from the background produces the additional left shadow effect. $\mathrm{B}_{2}$ (non moving target) is correctly matched but being closed the background, no shadow effect is observed. $\mathrm{B}_{3}$ (normal speed target) is half matched and produces a double shadow effect for the same reasons as $B_{1}$

\section{Segmentation}

The bee segmentation step takes advantage of the 3D information available. A color motion mask and depth mask are combined using adaptive thresholding to detect walking bees and flying bees. Then centroids $(\mathrm{X}, \mathrm{Y})$ of every region define the $2 \mathrm{D}$ position of the targets. The 3 rd coordinate $\mathrm{Z}$ (depth value) of the target is the median value of the corresponding region on the depth map.

\subsection{Color Motion Mask}

In [4] and [8], bees are detected by pattern matching using respectively eigenbee and oval graduate template over a background subtraction. A comparative study of advanced background subtraction methods is done in [13]. To succeed in outdoor conditions (e.g. unstable light, grass moving with wind), background subtraction methods need to be performed on a small temporal window. Problems arise for non-moving targets like fanning bees. We prefer to use a less computationally demanding temporally local method:

$$
\text { cMask }=\left(\left|I_{t-1}-I_{t}\right|>s\right) \&\left(\left|I_{t+1}-I_{t}\right|>s\right)
$$


where $I_{t}$ is the image at time $t$ and $s$ is the motion detection threshold. Low thresholds enable small changes detection (e.g. fanning bees that are constantly flapping wings), and high thresholds just detect flying and walking bees. The hue could be used to filter bee shadows, but it tends to overestimate motions when the saturation has a low value. Therefore a combined hue and gray mask is preferable. Filtering and morphological operation are applied to the motion mask to filter the noise.

\subsection{Depth Mask}

Flying bees produce either a local distance peak or depth shadow areas (e.g. matching failure due to speed) on the depth map. As explained in Section 2.3, high bees also produce a second depth shadow area on the same horizontal line due to background mismatching. The flying mask $d M a s k F$ is obtained by:

$$
d M a s k F=\left(D_{t}+b<D_{m}\right) \|\left(D_{t}=0 \& D_{m}=0\right)
$$

where $D_{t}$ is the depth map at time $t, D_{m}$ is the median depth map over a time period and $\mathrm{b}$ a depth detection threshold. Afterwards, morphological opening is applied to the mask.

Walking bees produce mainly a distance peak that is more or less observable according to quality of the background matching. The walking mask $d M a s k W$ is obtained by applying following operations: blurring, morphological erosion and peak detection.

\section{Tracking}

We use the widely spread GNN association method summarized in [14] based on a Kalman Filter with a constant speed model for tracking. Our GNN chooses the best association hypothesis between tracks and observation according to their probabilities using the Hungarian Algorithm. The following statistical distance is used:

$$
d_{i j}^{2}=\left(Y-M X^{+}\right)^{\prime} S^{-1}\left(Y-M X^{+}\right)
$$

It represents the association cost between the predicted position $\mathrm{i}$ and the observation $\mathrm{j}$, where $\mathrm{Y}$ is the observation, $\mathrm{M}$ the measurement matrix, $\mathrm{X}^{+}$the predicted position a priori and $\mathrm{S}$ the innovation covariance defined by $S=M E^{+} M^{\prime}+E m$ with $E m$ the measure noise matrix and $E^{+}$the predicted noise covariance matrix. The costs of new targets and false alarms are linked to the probability density functions $\beta_{\mathrm{NT}}$ and $\beta_{\mathrm{FA}}$. Associated observations are processed using target's Kalman Filter, and non-associated observations become candidates for a new track.

Kalman filters are tuned as followed: The initial noise matrix has to be especially high in order to handle high speed target entering the screen. As bees' motions seem chaotic outside the beehive, the model noise matrix also needs to be very high. In opposite, measures are relatively accurate (expect in case of merged observations) so the measure noise matrix could be low. 
With camera captured data, bee coordinates $(\mathrm{X}, \mathrm{Y})$ and $\mathrm{Z}$ are respectively pixels in the image reference and millimeters in the camera based reference. A projection of $\mathrm{X}$ and $\mathrm{Y}$ can be done using the intrinsic camera parameters :

$$
\operatorname{pos} X Y=F(\operatorname{cam} X Y-c X Y) Z
$$

where $\mathrm{cXY}$ is the projected optical center of the camera and $\mathrm{F}$ the focal distance. When measure of $\mathrm{Z}$ is unavailable (depth shadow effect), we take the predicted $\mathrm{Z}$ from the Kalman filter (state and error covariance) directly to the next prediction step, so the correction step is skipped for $\mathrm{Z}$.

\section{Preliminary Results}

A ground truth is needed to evaluate the results for counting and tracking. Labeling video frames over a long period of time is not feasible especially in cluttered situations. Moreover manual labeling is source of error, when estimating counting error rate of counters, human counting error is usually taken in account in the estimation. To get around those problems, we made a simulator that produces comparable honeybee trajectories and corresponding color images and depth images as the $3 \mathrm{D}$ camera would capture. Compared to labeled data, simulated data has the advantage to be scalable and easily tunable.

Simulated bees tend to reach a random goal which changes time to time. A constant speed motion model is used and noise is injected to randomize the trajectories. Shown in Figure 6, the color image is simply built up using bee images well resized according to its 3D position $(\mathrm{X}, \mathrm{Y}, \mathrm{Z})$. The depth image is made up following the 3D camera shadow rules. For example, if the bee is far away from the ground, the matching between left and right frame can not be done for the background behind the bee that is observable on the image. In this case the bee produces a depth shadow area on its side. Then the blur effect due to the speed or of wings flapping could also result in a non-matching area as well.

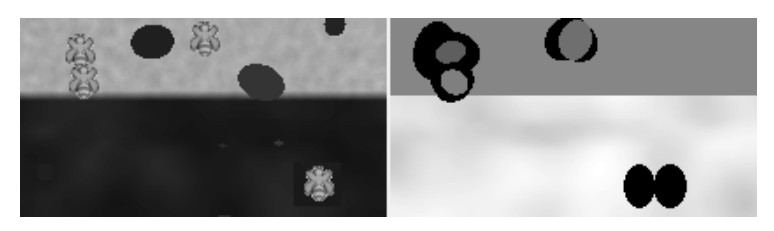

Fig. 6. Images and depth map produced by the simulator

The following results are preliminary, and conditions for the real captured data were absolutely uncontrolled. For example, some plant's stem could exceed the height of the flight board resulting in more false alarms. Bees half positioned in the entrance of the beehive are left apart in the statistics. They don't exhibit any interesting behavior and their detection is even manually difficult. 


\subsection{D Bee Detection}

This part shows the performance of the 3D bee detection step. It especially witnesses the proportion of hazardous depth detection for overlapping bees. Miss detected bees is not a main issue since no counting step is done on every frame in a absolute manner. It will only result in no association during one or few tracking steps. Following figures are an average number of bee per frame over a sequence of frame.

Simulated: Over 200 simulated frames with a constant number of 15 moving bees, 0.5 bee per frame are over detected and the $\mathrm{Z}$ coordinate is not well detected on 1.7 bee.

Real data: Over 10 frames picked up randomly from a captured video. In average, a frame contains around 13 targets, 2.7 bees per frame are over detected and the $\mathrm{Z}$ coordinate is not well detected on 2.3 bees.

\subsection{D Tracking}

This part shows the performance of the tracking step taking the advantage of the $3 \mathrm{D}$ information.

Simulated data: Table 3 shows the advantage of having 3D information for a target with an high occluding rate. Results have been established using the measures from simulated trajectories over 500 frames on 50 frames track lengths. For small amounts of bees, better 2D tracking results are explained by the more restrictive gating step in $3 \mathrm{D}$ than in 2D. Under real conditions, 3D information is not constantly available so we can expect lower results. But the 2D less accurate measures would also make the 2D tracking maintenance rate decrease.

Real data: Since no trajectory ground truth has been established, it is difficult to estimate the results of tracking. We estimate that $80 \%$ of the bees are well tracked. The $20 \%$ failure is mainly is due to high speed targets, long miss detection, complex scenarios (e.g. triple crossing).

Table 3. Comparative track maintenance rates between 2D or 3D tracking on simulated observations. Tracks represent the number of track generated over the 500 frames. Bees represents the number of bees flying simultaneously in the area

\begin{tabular}{|l|c|c|c|c|c|}
\hline Bees(tracks) & $2(18)$ & $5(50)$ & $10(89)$ & $20(157)$ & $30(236)$ \\
\hline 2D tracking & $100 \%$ & $98 \%$ & $88 \%$ & $88 \%$ & $78 \%$ \\
\hline 3D tracking & $94 \%$ & $95 \%$ & $96 \%$ & $93 \%$ & $91 \%$ \\
\hline
\end{tabular}

\section{Conclusion}

Our work takes place in the context of an environment monitoring platform. The purpose of the project is to analyse the behavior of bees using to their trajectories and other data provided by the platform. This article positions the base for a 3D monitoring system at the beehive entrance including: 3D capture, bee detection and tracking. Detection and tracking algorithms are still in a early state, but the preliminary results and following perspectives are promising. 


\subsection{Perspectives}

In complex tracking situations (e.g. $\mathrm{Z}$ info missing, 3 bees overlapping), our GNN method reaches its limit. We are currently working to use a combined MHT-IMM tracking method to improve tracking results.

This work is a preliminary for a behavioral analysis. Based on 3D trajectories collected, we will be able to build a database of bees' behaviors of front at the beehive entrance. To ensure the feasibility of behavior identification of bees outside the beehive, we will implement a behavior recognition system based on HMM as the image of [5]. Then as [8] or [6], we plan to take advantage of the behavior knowledge to improve the tracking step. The first idea is to drive the choice of the motion model in IMM. To go further, we also plan to use data coming from the platform such as weather, historical behaviors and even crossed data between beehives.

\section{References}

1. Chauvin, R.: Sur la mesure de l'activité des abeilles au trou de vol d'une ruche a dix cadres. Insectes Sociaux 23 (1976) 75-81

2. Struye, M., Mortier, H., Arnold, G., Miniggio, C., Borneck, R.: Microprocessor-controlled monitoring of honeybee flight activity at the hive entrance. Apidologie 25 (1994) 384-395

3. Streit, S., Bock, F., Pirk, C., Tautz, J.: Automatic life-long monitoring of individual insect behaviour now possible. Zoology 106 (2003) 169-171

4. Khan, Z., Balch, T., Dellaert, F.: A rao-blackwellized particle filter for eigentracking. In: Conference on Computer Vision and Pattern Recognition (CVPR). Volume 2., IEEE (2004) II-980

5. Feldman, A., Balch, T.: Representing honey bee behavior for recognition using human trainable models. Adaptive Behavior 12 (2004) 241-250

6. Veeraraghavan, A., Chellappa, R., Srinivasan, M.: Shape-and-behavior encoded tracking of bee dances. Transactions on Pattern Analysis and Machine Intelligence (PAMI) 30 (2008) 463-476

7. Maitra, P., Schneider, S., Shin, M.: Robust bee tracking with adaptive appearance template and geometry-constrained resampling. In: Workshop on Applications of Computer Vision (WACV), IEEE (2009) 1-6

8. Campbell, J., Mummert, L., Sukthankar, R.: Video monitoring of honey bee colonies at the hive entrance. Visual observation \& analysis of animal \& insect behavior (ICPR) (2008)

9. Estivill-Castro, V., Lattin, D., Suraweera, F., Vithanage, V.: Tracking bees-a 3d, outdoor small object environment. In: Conference on Image Processing (ICIP), IEEE (2003) 10211024

10. Blois, J.: Vidéosurveillance d'abeilles, comptage d'entrées/sorties à l'entrée de la ruche. (2011)

11. Nummiaro, K., Koller-Meier, E., Svoboda, T., Roth, D., Van Gool, L.: Color-based object tracking in multi-camera environments. Lecture Notes in Computer Science (2003) 591-599

12. Piatti, D.: Time-of-Flight cameras: test, calibration and multi-frame registration for automatic 3D object reconstruction. PhD thesis, Politecnico di Torino, Italy (2011)

13. Parks, D., Fels, S.: Evaluation of background subtraction algorithms with post-processing In: Conference on Advanced Video and Signal Based Surveillance (AVSS), IEEE (2008) 192-199

14. Blackman, S., Popoli, R.: Design and analysis of modern tracking systems. Volume 685. Artech House Norwood, MA (1999) 